\title{
Updated guidelines for the management of thyroid disorders in pregnancy
}

A new set of guidelines from the Endocrine Society on the management of thyroid dysfunction during pregnancy has been published. The impetus for updating the 2007 guidelines relates to morerecent data on the "measurement of free $\mathrm{T}_{4}$ in pregnancy, use of propylthiouracil, diagnosis of fetal hyperthyroidism, need for increased iodine in pregnancy and need for early recognition of maternal

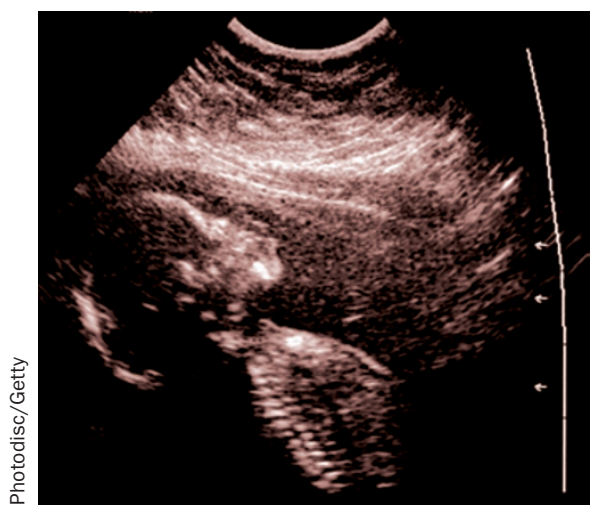

hypothyroidism," explains Leslie De Groot, Chair of the task force charged with updating the recommendations.

In response to FDA warnings on the effects of propylthiouracil and methimazole during pregnancy, the task force members recommend that clinicians switch patients with maternal hyperthyroidism from propylthiouracil to methimazole therapy after the end of the first trimester. This switch aims to minimize both the possible risk of congenital abnormalities associated with the use of methimazole during the first trimester and the rare risk of severe liver toxicity associated with the use of propylthiouracil.

Owing to problems with the accuracy of some free $\mathrm{T}_{4}$ assays during pregnancy, the task force recommends the establishment of laboratory-specific, trimester-specific reference ranges of free $\mathrm{T}_{4}$ levels or the use of alternative approaches, such as adjusted $\mathrm{T}_{4}$ measurements.
Universal screening of healthy women for thyroid dysfunction before pregnancy was not recommended, but the task force members unanimously recommend targeted screening of high-risk women during the prenatal period. Members were split with regards to whether all newly pregnant women should undergo screening, with some recommending universal screening but the majority being neither for nor against, owing to insufficient evidencebased data showing a benefit in treating maternal subclinical hypothyroidism.

Other changes to the guidelines relate to iodine nutrition and timing and indications for measurement of thyroid autoantibodies during pregnancy.

Carol Wilson

Original article De Groot, L. et al. Management of thyroid dysfunction during pregnancy and postpartum: an Endocrine Society clinical practice guideline. J. Clin. Endocrinol. Metab. 97, 2543-2565 (2012) 